Artigo / Article

\title{
Determinação da acurácia do método qualitativo da medida da atividade da glicose-6-fosfato desidrogenase
}

\author{
Determination of the accuracy of the measurement method for dehydrogenase activity
}

Letícia L. Giovelli ${ }^{1}$

Suzane Dal Bó

Raquel Weber ${ }^{3}$

Ana Paula Santin ${ }^{3}$

Simone M. Castro ${ }^{4}$

\begin{abstract}
A deficiência de glicose-6-fosfato desidrogenase (G6PD) é um problema de saúde pública que afeta aproximadamente 400 milhões de pessoas no mundo. No mercado, existem vários métodos que medem a atividade da G6PD. Os objetivos deste estudo foram determinar a acurácia do método de Brewer frente a um padrão de referência e estimar a prevalência de deficiência de G6PD na amostra. Foi realizado um estudo transversal de grupo de pacientes internados no HCPA com icterícia a esclarecer, no período de junho de 2004 a maio de 2005. Amostras foram processadas pelo método de Brewer e pelo método de Normalização da Hemoglobina, o qual foi usado como padrão ouro. Foi analisado para atividade da G6PD um total de 173 pacientes. A idade variou de 1 dia a 82 anos, sendo que 66\% da amostra possuía até 15 dias de vida. A

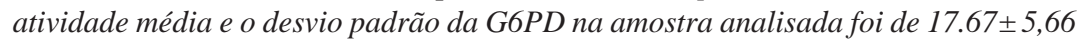
U/gHb. A freqüência estimada, pelo padrão ouro, da deficiência de G6PD, foi de 13 (7,7\%) pacientes com deficiência parcial ou total, e pelo método de Brewer foi de 14 (8,67\%). A sensibilidade do método de Brewer comparada com o método quantitativo da Normalização da Hemoglobina foi de 92,8\% e a especificidade foi de 98,7\%. A deficiência de G6PD é prevalente em nosso meio. Testes de baixo custo, tais como o teste de Brewer, podem ser utilizados como testes de triagem desta deficiência, principalmente no monitoramento de recém-nascidos que estão sob o risco de desenvolver icterícia neonatal. Rev. bras. hematol. hemoter. 2007;29(4):378-381.
\end{abstract}

Palavras-chave: G6PD; sensibilidade; especificidade; método de Brewer; icterícia.

\section{Introdução}

A deficiência de glicose-6-fosfato desidrogenase (G6PD) é uma desordem genética que afeta aproximadamente 400 milhões de pessoas no mundo. Atinge especialmente as regiões mediterrâneas, africanas e asiáticas. Cerca de $7,5 \%$ da população carrega um ou mais genes dessa condição. Atualmente já foram descritas mais de 140 mutações envolvendo os 12 éxons codificadores e cerca de 400 enzimas variantes. ${ }^{4,12,16} \mathrm{O}$ gene que codifica a G6PD se localiza no cromossomo $\mathrm{X}$, sendo a deficiência mais freqüente no sexo masculino. No sexo feminino, a deficiência pode ser expressa na forma homozigota ou heterozigota. ${ }^{12}$

A deficiência de G6PD é a alteração enzimática hereditária mais comumente associada à anemia hemolítica, principalmente por exposição do indivíduo deficiente a agentes oxidantes. ${ }^{7}$ As manifestações clínicas associadas com a deficiência de G6PD são: hemólise induzida por drogas, hemólise induzida por infecções, favismo, icterícia neonatal e anemia hemolítica crônica não esferocítica. ${ }^{18}$

As icterícias hemolíticas ocorrem com maior freqüência depois da icterícia fisiológica. Elas são agrupadas em três grandes categorias: imunes, hereditárias e adquiridas. A deficiência de glicose-6-fosfato desidrogenase (G6PD) é

${ }^{1}$ Curso de Especialização em Análises Clínicas da UFRGS,

${ }^{2}$ Laboratório de Hematologia do Serviço de Patologia Clínica do HCPA.

${ }^{3}$ Faculdade de Farmácia, UFRGS.

${ }^{4}$ Professora da Disciplina de Análises Hematológicas, Faculdade de Farmácia, UFRGS.

Correspondência: Simone Martins de Castro

Faculdade de Farmácia - Universidade Federal do Rio Grande do Sul

Avenida Ipiranga, 2752 - sala 303

90610-000 - Porto Alegre-RS - Brasil

E-mail: scastro@adufrgs.ufrgs.br 
a alteração enzimática hereditária mais comumente associada à anemia hemolítica, principalmente por exposição do indivíduo deficiente a agentes oxidantes. ${ }^{7}$

O objetivo principal do tratamento da icterícia é evitar a encefalopatia bilirrubínica aguda e crônica ou kernicterus, termos utilizados para designar a disfunção neurológica induzida pela bilirrubina (BIND). Esta patologia é causada em grande parte pela passagem de bilirrubina indireta livre através da barreira hemato-encefálica e sua deposição no cérebro, causando danos irreversíveis às células, podendo levar a distúrbios auditivos, visuais e cognitivos, além de retardo mental e até mesmo à morte. ${ }^{5,10}$ Relatos da literatura demonstram que $71 \%$ dos pacientes com kernicterus possuem deficiência de G6PD. ${ }^{13}$

Vários são os métodos descritos na literatura para a determinação da atividade da G6PD, incluindo métodos qualitativos, quantitativos e moleculares. ${ }^{15,18}$ Estes métodos apresentam diferentes graus de sensibilidade e especificidade, assim como diferentes formas de apresentação dos resultados. Contudo, a grande maioria dos métodos disponíveis é capaz de detectar deficiências totais, mas falha na detecção de casos de deficiência parcial. Torna-se de grande importância que o laboratório conheça a acurácia do método por ele selecionado, para obter resultados de alta confiabilidade. Os objetivos deste estudo foram determinar a especificidade e sensibilidade do teste de Brewer usando-se o teste quantitativo de normalização da hemoglobina para a determinação da atividade da G6PD como padrão ouro e estimar a prevalência de deficiência de G6PD na amostra analisada.

\section{Casuística e Métodos}

\section{Amostras}

Foi realizado um estudo transversal de grupo de 173 pacientes com icterícia a esclarecer, internados em diferentes serviços do Hospital de Clínicas de Porto Alegre (HCPA), no período de junho de 2004 a maio de 2005.

\section{Métodos}

Amostras de sangue total coletadas com EDTA foram processadas pelo teste de redução da meta-hemoglobina (método de Brewer) no Setor de Hematologia do Laboratório de Patologia Clínica do HCPA. Nas mesmas amostras foi quantificada a atividade da G6PD pelo método de Normalização da Hemoglobina no Laboratório de Análises Clínicas e Toxicológicas da Faculdade de Farmácia da Universidade Federal do Rio Grande do Sul. Este segundo método foi usado como padrão ouro. O projeto foi submetido e aprovado pelo Comitê de Ética do HCPA. Brewer):

Teste da redução da meta-hemoglobina (Método de

A $200 \mu \mathrm{L}$ de sangue com EDTA foram adicionados $10 \mu \mathrm{L}$ de nitrito de sódio-glicose e $10 \mu \mathrm{L}$ de solução de azul de metileno. Após incubação em banho-maria a $37^{\circ} \mathrm{C}$ durante três horas, foi transferido $1 \mathrm{~mL}$ desta solução para um tubo contendo $10 \mathrm{~mL}$ de água tipo 2 e misturou-se por inversão. Por leitura visual, comparou-se a cor formada no tubo da amostra com as cores dos tubos controles positivo e negativo. Para uma atividade normal de G6PD, a reação final deve apresentar a coloração vermelho vivo, uma vez que a solução de nitrito transforma a hemoglobina em meta-hemoglobina, e esta é reconvertida em hemoglobina na presença de azul de metileno. Na presença de uma atividade reduzida de G6PD, a coloração final é castanha, pois ocorre a formação de meta-hemoglobina sem reconversão. ${ }^{3}$

\section{Determinação da atividade enzimática da G6PD (Intercientífica) \\ Para a dosagem da atividade enzimática da G6PD pelo} método quantitativo utilizou-se o kit Neolisa G-6-PD (Interscientific Corporation, 2700 North 29th Avenue - Suite 220, Hollywood, FL - USA Cat. Nr 3570-050) que emprega o procedimento de Normalização da Hemoglobina. ${ }^{13} \mathrm{O}$ kit selecionado oferece uma medida rápida da atividade da G6PD contida na amostra, expressando os resultados diretamente em unidades por grama de hemoglobina (U/gHb). Foram utilizados controles normal, intermediário e deficiente fornecidos pela empresa Intercientífica.

Descrição breve: Foram pipetados $5 \mu \mathrm{L}$ de amostra de sangue total e controles em microplacas de microtitulação com fundo em "U". A estes foram adicionados $75 \mu \mathrm{L}$ de reagente de eluição e agitados em um agitador orbital por 20 minutos. Quinze $\mu \mathrm{L}$ do eluído foram transferidos para outra microplaca de fundo chato juntamente com $75 \mu \mathrm{L}$ de reagente de trabalho e agitados durante 10 minutos. Uma primeira leitura em 405nm foi realizada para medida da concentração de hemoglobina das amostras e dos controles. Posteriormente foram adicionados $100 \mu \mathrm{L}$ do reagente de cor, e efetuada uma segunda leitura, em 550nm, em modo cinético. As alterações na densidade óptica por minuto foram registradas em 15 medidas tomadas no intervalo de 1 minuto.

\section{Análise estatística}

Para análise estatística foram utilizadas análise de freqüência, variância de grupos e Teste t, com significância P 0,05 e utilizando o SPSS software, versão 12.0.

\section{Resultados}

Foi determinada a atividade da G6PD, um total de 173 pacientes com icterícia a esclarecer, sendo 72 (49,7\%) do sexo feminino e 73 (50,3\%) do masculino. A idade variou de 1 dia a 82 anos, sendo que $66 \%$ dos pacientes possuíam até 15 dias de vida.

Os valores de referência para a atividade da G6PD, utilizados neste estudo, variaram de $\leq 2,6 \mathrm{U} / \mathrm{gHb}$ para pacientes deficientes, entre 2,6 - 7,7 U/gHb para parcialmente deficien- 
tes e pacientes normais com atividade $>$ 7,7 U/gHb. Estes valores foram previamente estabelecidos pelo laboratório em uma amostra normal.

Nossos resultados demonstraram uma distribuição gaussiana dos valores da atividade de G6PD. A atividade média da G6PD na amostra analisada foi de 17,67 U/gHb, com um desvio padrão de 5,66 U/gHb, como demonstrado na Figura 1.

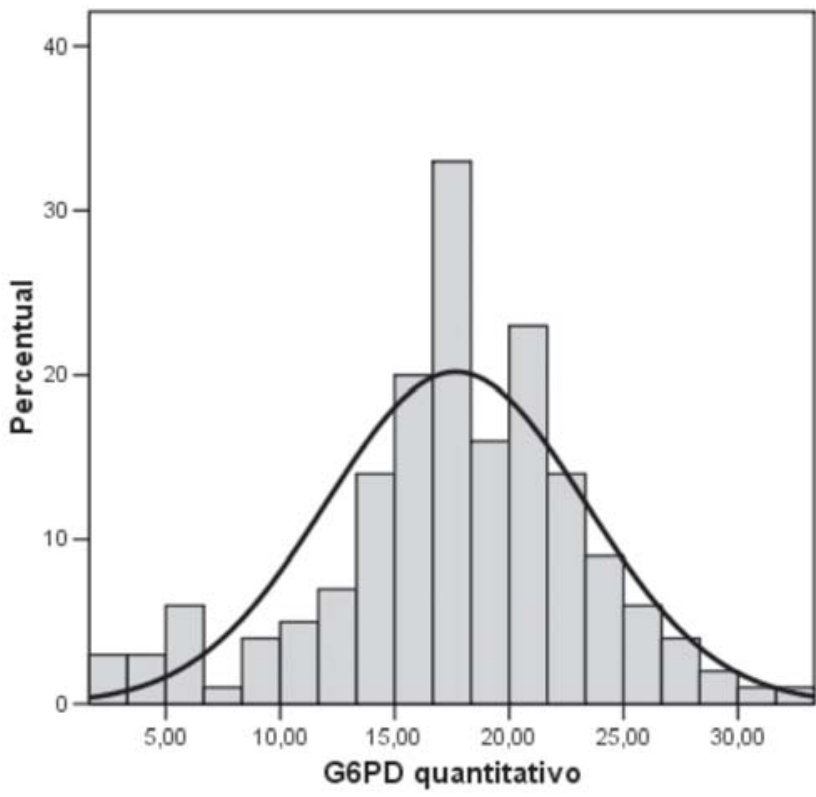

Figura 1. Distribuição dos valores da atividade de G6PD (U/gHb) de 173 pacientes do HCPA com icterícia a esclarecer

Tabela 1. Resultados da atividade enzimática da G6PD nos pacientes deficientes e normais pelos métodos de Brewer e de normalização da hemoglobina

\begin{tabular}{ccc}
\hline Atividade G6PD & \multicolumn{2}{c}{$\begin{array}{c}\text { Método de Normalização da } \\
\text { Hemoglobina }\end{array}$} \\
\hline Método de Brewer & $\begin{array}{c}\text { Deficiente } \\
(\leq 7,7 \mathrm{U} / \mathrm{gHb})\end{array}$ & $\begin{array}{c}\text { Normal } \\
(>7,7 \mathrm{U} / \mathrm{gHb})\end{array}$ \\
\hline Deficiente & 13 & 2 \\
Normal & 1 & 157 \\
\hline Total & 14 & 159 \\
\hline
\end{tabular}

$\mathrm{Hb}=$ Hemoglobina

A freqüência de deficiência de G6PD estimada, pelo método de quantificação da atividade enzimática, na amostra foi de 14 (8,1\%) pacientes com deficiência parcial ou total, e pelo teste de Brewer foi de 15 (8,67\%)

A sensibilidade do método de Brewer comparado com o método quantitativo da Normalização da Hemoglobina foi de 92,8\%. Já a especificidade foi de 98,7\%. O valor preditivo positivo foi $86,7 \%$ e o valor preditivo negativo foi $99 \%$ (Tabela 1).

\section{Discussão}

A deficiência de G6PD é a deficiência enzimática congênita mais comum em humanos, presente em 400 milhões de pessoas em todo o mundo., ${ }^{9,11,16}$ A prevalência da deficiência de G6PD total e parcial encontrada neste estudo foi de $8,67 \%$ em pacientes com icterícia a esclarecer. Outros estudos realizados em diferentes regiões do Brasil constataram prevalências em torno de $10 \%$ entre os homens de ascendência africana. Já entre os homens euro-descendentes dos estados de São Paulo e do Rio Grande do Sul foram encontradas freqüências de deficientes de G6PD em torno de $1 \%$ a $6 \%$. ${ }^{2,6,8,19,20}$

Para a detecção dessa desordem genética, testes de triagem qualitativos e quantitativos têm sido desenvolvidos. ${ }^{9}$ Os testes qualitativos, tais como o teste de Brewer, têm sido largamente utilizados como teste de triagem, pelo baixo custo, pela simplicidade e rapidez de execução. ${ }^{18}$ A determinação quantitativa da atividade de G6PD é um método mais preciso para a detecção de pacientes deficientes, ${ }^{15}$ principalmente na caracterização de indivíduos com mutações que causam deficiências menos severas.

Uma vez que dispomos de vários testes disponíveis no mercado, torna-se importante avaliar a acurácia e limitações dos métodos selecionados pelos laboratórios de análises clínicas.

Nossos resultados demonstraram que o teste de Brewer é um bom teste de screening para detectar pacientes deficientes da atividade enzimática da G6PD por apresentar um valor de sensibilidade de 92,8\% e especificidade de $98,7 \%$. Entretanto, essa técnica possui algumas limitações, sendo a principal delas o fato de não detectar heterozigotos. Por muitos anos, pacientes heterozigotos não eram considerados sob risco. Segundo Kaplan, ${ }^{11}$ e Krzelj, ${ }^{14}$ pacientes heterozigotos estão sob o mesmo risco de desenvolver icterícia neonatal que pacientes homozigotos.

O método de Brewer apresentou um valor preditivo positivo baixo (86,7\%). A inacurácia do teste, demonstrada pela detecção de duas amostras falso positivas, é provável por outra limitação do método. Das 173 amostras de pacientes com icterícia a esclarecer, $66 \%$ eram compostas por recém-nascidos com até 15 dias de vida. Há relatos na literatura que demonstram que o método de Brewer em casos de pacientes com bilirrubina elevada é limitado. Este fenômeno poderia ser causado pela interferência da bilirrubina na redução da meta-hemoglobina, por diferentes mecanismos de stress oxidativo nos neonatos ou simplesmente pela interferência da cor da bilirrubina na leitura subjetiva da mudança de cor do teste. ${ }^{18}$

A possibilidade de casos de deficientes de G6PD poderem manifestar anemia hemolítica aguda importante, até mesmo fatal, na presença de fatores oxidantes fortes, e a associação entre a deficiência de G6PD e a icterícia neonatal, ${ }^{8}$ justificam a importância da detecção e da orientação preventiva 
dos enzimopênicos nas populações, sobretudo naquelas em que a probabilidade de encontro da variante Mediterrânea, mais grave, for maior.

A deficiência de G6PD é prevalente em nosso meio. Casos de encefalopatia bilirrubínica causados por deficiência de G6PD podem ser evitados, desde que esta deficência seja identificada e tratada precocemente. Testes de baixo custo, tais como o teste de Brewer, podem ser utilizados como testes de screening desta deficiência, principalmente no monitoramento de recém-nascidos que estão sob o risco de desenvolverem hiperbilirrubinemia.

Técnicas de medida da atividade enzimática e estudos moleculares podem aumentar a eficácia dos testes, para fins confirmatórios, podendo ser usadas como uma ferramenta complementar, informando aos médicos dados mais conclusivos.

\section{Abstract}

Glucose-6-phosphate dehydrogenase deficiency (G6PD) is a public health problem which affects about 400 millions of people all over the world. Some methods that measure the activity of G6PD have already been developed. Thus, the aim of this study was to evaluate the accuracy of the Brewer's method compared with a standard reference and estimate the prevalence of G6PD deficiency in the sample. A cross-sectional study of a group of patients in HCPA presenting with jaundice was carried out from June 2004 to May 2005. Samples were processed by the metahaemoglobin reduction test (Brewer's method) and by the method of Haemoglobin Normalization, which was used as the standard reference. A total of 173 patients were analyzed for G6PD activity. The ages varied from one day to 82 years old with 66\% of the sample being less than 16 days old. The mean activity and standard deviation of G6PD for the analyzed sample was $17.67 \pm 5.66 \mathrm{U} / \mathrm{gHb}$. The estimated frequencies of G6PD deficiency for the standard reference and Brewer's method were $13(7.7 \%)$ subjects (total or partial deficiency) and 14 (8.67\%), respectively. When the Brewer's method was compared with the quantitative method of Hemoglobin Normalization, it showed a sensitivity of $92.8 \%$ and specificity of $98.7 \%$. As G6PD deficiency predominates in our society, low cost tests, such as the Brewer's test can be used for screening this deficiency, mainly to monitor newborn babies who are at risk of developing jaundice. Rev. bras. hematol. hemoter. 2007;29(4):378-381.

Key words: G6PD; sensitivity; specificity; Brewer's method; jaundice.

\section{Referências Bibliográficas}

1. Atay E, Bozaykut A, Ipek I. Glucose-6-phosphate dehydrogenase deficiency in neonatal indirect hyperbilirrubinemia. Journal of Tropical Pediatrics. 2006;52:1:56-8.

2. Azevêdo Es, Azevedo TFS. Glucose-6-phosphate dehydrogenase deficiency and neonatal jaundice in Bahia, Brazil. Cienc Cult. 1974; 26(1):44-7.

3. Beiguelmas B. Genética Médica. $3^{\circ}$ Ed. São Paulo: Edart, 1979.

4. Beutler E. Discrepancies between genotype and phenotype in hematology: an important frontier. Blood. 2001;98:9:2597-602.
5.Bhutani VK,Johnson LH, Shapiro SM. Kernicterus in sick and preterm infants (1999-2002): a need for an effective preventive approach. Semin Perinatol. 2004;28:5:319-25.

6. Compri MB, Saad ST, Ramalho AS. Genetico-epidemiological and molecular investigation of G-6-PD deficiency in a Brazilian community. Cad Saúde Publica. 2000;16:335-42.

7. Dhaliwal G, Cornett PA, Tierney LM Jr. Hemolytic anemia. Am Fam Physician. 2004;1:69:11:2599-606.

8. Garlipp CS, Ramalho AS. Aspectos clínicos e laboratoriais da deficiência de desidrogenase de 6-fosfato de glicose (G-6-PD) em recém-nascidos brasileiros. Rev Brasil Genet. 1988;11:717-28.

9. Gurbuz N, Aksu TA, Van Noorden CJ. Biochemical and cytochemical evaluation of heterozygote individuals with glucose6-phosphate dehydrogenase deficiency. Acta histochemica. 2005;107:4:261-7.

10. Johnson LH, Bhutani VK, Brown AK. System-based approach to management of neonatal jaundice and prevention of kernicterus. J Pediatr 2002;140:4:396-403.

11. Kaplan M, Beutler E, Vreman H, Hammerman C, Levy-Lahad E, Renbaum P, et al. Neonatal hyperbilirrubinemia in glucose-6phosphate dehydrogenase-deficient heterozygotes. Pediatrics 1999;1:104:68-74.

12. Kaplan M, Hammerman C. Glucose-6-phosphate dehydrogenase deficiency: a hidden risk for kernicterus. Semin Perinatol 1996; 28:5:356-64.

13. Kaplan M, Hammerman C. Understanding severe hyperbilirubinemia and preventing kernicterus: adjuncts in the interpretation of neonatal serum bilirubin. Clin Chim Acta. 2005; 356:1-2:9-21.

14. Krzelj V, Zlodre S, Terzic J, Mestrovic M, Jaksic J, Pavlov N. Prevalence of G-6-PD deficiency in the Croatian Adriatic Coast Population. Arch Med Res. 2001;32:5:454- 7.

15. Lin Z, Fontaine JM, Freer DE, Naylor EW. Alternative DNAbased newborn screening for glucose-6-phosphate dehydrogenase deficiency. Mol Genet Metab. 2005;86:1-2:212-9.

16. Nicol CJ, Zielenski J, Tsui LC, Wells PG. An embryoprotective role for glucose-6-phosphate dehydrogenase in developmental oxidative stress and chemical teratogenesis. The FASEB Journal. 2000;14:1:111-27.

17. Reclos GJ, Hatzidakis CJ, Schulpis KH. Glucose-6-phosphate dehydrogenase deficiency neonatal screening: preliminary evidence that a high percentage of partially deficient female neonates are missed during routine screening. J Med Screen. 2000; $7(1): 46-51$.

18. Sampavat S, Kittikalayawong A, Nuchprayoon I, Ungbumnet W. The value of methemoglobin reduction test as a screening test for neonatal glucose-6-phosphate dehydrogenase deficiency. J. Med. Assoc. Thai. 2001;1:84:S91-S98.

19. Weimer TA, Salzano FM, Westwood B, Beutler E. G6PD variants in three South American ethnic groups: population distribution and description of two new mutations. Hum Hered. 1998; 48: 2:92-6.

20.Weimer TA, Salzano FM, Westwood B, Beutler E. Molecular characterization of glucose-6-phosphate dehydrogenase variants from Brazil. Hum Biol. 1993; 65:1:41-7.

Avaliação: Editor e dois revisores externos

Conflito de interesse: não declarado.

Recebido: 24/05/2006

Aceito após modificações: 25/06/2007 